\title{
Influencia de la reserva cognitiva en la calidad de vida en sujetos con enfermedad de Alzheimer
}

\author{
Juan L. Sánchez-Rodríguez ${ }^{1 *}$, Clara Torrellas-Morales ${ }^{1}$, María J. Fernández-Gómez ${ }^{2}$ y Javier Martín-Vallejo ${ }^{2}$ \\ 1 Departamento de Psicologia Básica, Psicobiologia y Metodología de las C.C. Facultad de Psicología. Universidad de Salamanca \\ 2 Departamento de Estadística. Facultad de Medicina. Universidad de Salamanca
}

\begin{abstract}
Resumen: La calidad de vida (CV) se define como la percepción personal que un individuo tiene de su situación vital. Dentro de los factores que pueden influir en la CV, se encuentra la Reserva Cognitiva (RC), que podría entenderse como la capacidad del cerebro para hacer frente al daño cerebral generado por la patología, mediante procesos cognitivos preexistentes o compensatorios. El objetivo principal de este estudio, consiste en analizar, como influye la $\mathrm{RC}$ en la auto-percepción subjetiva de la $\mathrm{CV}$ en sujetos diagnosticados de Enfermedad de Alzheimer (EA) y comprobar si existen perfiles diferenciales en función de la sintomatología depresiva y el estado cognitivo de los mismos. La muestra utilizada estaba formada por 112 sujetos que se distribuyeron en dos grupos: uno de 74 sujetos diagnosticados de EA, y otro de 38 sujetos sanos. Se ha utilizado el cuestionario SF-36 para evaluar la CV. En relación a la variable RC, destacar que los sujetos con mayor RC, puntuaron más alto en cada una de las dimensiones del SF36. La RC podría ser una fuente de influencia en la percepción de la CV de las personas con EA, en la medida en que sus diversos componentes conducirían a la consecución de una capacidad funcional más óptima y una aceptación del estado cognitivo.

Palabras clave: Calidad de vida; demencia; enfermedad de Alzheimer; estado de salud; reserva cognitiva; autovaloración.
\end{abstract}

Title: Influence of cognitive reserve on quality of life of patients with Alzheimer's Disease.

Abstract: Quality of life (QL) is defined as the personal perception an individual has of his or her own life situation. Among the factors that can affect QL is Cognitive Reserve (CR) which can be understood as the brain's capacity to resist the brain damage caused by pathology through pre-existing or compensatory cognitive processes. The main objective of this study is to analyze how CR affects the subjective self-perception of QL in patients diagnosed with Alzheimer's Disease (AD) and to determine the existence of different profiles in terms of depressive symptoms and cognitive state. The sample comprised 112 individuals divided into two groups: one group with 74 patients diagnosed with $\mathrm{AD}$, and the other with 38 healthy participants. The SF-36 questionnaire was used to assess QL. As regards cognitive reserve, it was found that subjects with greater CR scored higher in each of the dimensions of the SF-36. CR could be a source of influence on perception of $\mathrm{QL}$ in persons with $\mathrm{AD}$, to the extent that its different components would lead to a more optimal functional capability and a better acceptance of one's cognitive state.

Key words: Quality of life; dementia; Alzheimer disease; health status; cognitive reserve; self-assessment.

\section{Introducción}

En la actualidad, estamos siendo testigos de un creciente envejecimiento de la población, lo que supone un incremento notable de enfermedades ligadas a la edad, como es el caso de las demencias (Rodríguez y Sánchez, 2004). En concreto, la enfermedad de Alzheimer (EA), es uno de los procesos más incapacitantes para las personas que lo padecen, ya que conlleva un deterioro cognitivo y una pérdida progresiva de habilidades, llegando a producir una reducción de las actividades placenteras y/o cotidianas, traduciéndose en una disminución de la calidad de vida (CV) (Almkvist y Winblad, 1999; Brod, Steward, Sands y Walton, 1999; Lawton, 1994; Lopera, 1997; Rabins y Black, 2007; Wierenga y Bond, 2007).

A pesar de la gran atención que ha recibido el concepto calidad de vida, todavía no se ha logrado una definición consensuada del mismo, dada su naturaleza multidimensional y la diversidad de variables por las que se ve influido (Zea Herrera et al., 2008). No obstante en 1994, la Organización Mundial de la Salud (OMS), llevó a cabo un intento de aproximación a dicho constructo, definiéndolo como una "Percepción personal que un individuo tiene de su situación de vida, dentro del contexto cultural y de los valores en los que vive, en relación con sus objetivos, expectativas, valores e intereses". A partir de ahí, numerosos autores han ido rea-

* Dirección para correspondencia [Correspondence address]: Juan Luis Sánchez Rodríguez. Facultad de Psicología. Avd/ La Merced s/n. 37005. Salamanca (España) E-mail: jlsanch@usal.es lizando matizaciones con respecto a dicha definición. Felce y Perry (1995), las han recogido en un modelo con cuatro concepciones diferentes de la CV. Así, según estos autores, la CV puede ser entendida, desde un punto de vista objetivo, como las condiciones vitales que acompañan a un individuo; desde una perspectiva subjetiva como la satisfacción con dichas condiciones de vida; desde una postura intermedia que incluye ambas opciones y finalmente desde una óptica más global en la que se tienen en cuenta ambas posturas y su interacción con el sistema de valores y expectativas del sujeto.

Otros autores (Lövdén, Ghisletta y Lindenberger, 2004; Revell, Caskie, Willis y Schaie, 2009; Whitehouse, 1999), han orientado su enfoque a la búsqueda de las dimensiones que conforman este constructo. Lawton propuso en 1994 un modelo que considera que la CV está integrada por tres factores: el físico, el psicológico y el social, siendo la salud física, el elemento más discriminativo de este constructo (Castellón y Romero, 2004; Lucas-Carrasco, Skevington, GomezBenito, Rejas y March, 2011; Oswald, 2004).

Por otra parte, autores como Brod et al. (1999); Smith et al. (2005), consideran que la aplicación práctica de este constructo al campo de las demencias, requiere tener en cuenta elementos adicionales como: las alteraciones cognitivas asociadas al trastorno, que pueden introducir sesgos tanto a nivel de percepción de la situación, como a nivel de comunicación de la misma (Baquero, 2009; Hoe, Hancock, Livingston y Orrell, 2009; Martínez-Martín, 2002); la progresiva dependencia funcional que provoca la enfermedad (Misso- 
tten et al., 2007); la existencia o no de una red social de apoyo y/o acceso a recursos asistenciales que faciliten el afrontamiento de los cambios asociados (Castellón, Gómez y Martos, 2004; Kitwood y Bredin, 1991; Molina, Meléndez y Navarro, 2008); la frecuencia con la que el bajo estado de ánimo y la ansiedad acompañan al deterioro (Hoe et al., 2009; Livingston, Cooper, Woods, Milne y Katona, 2008; Lucas-Carrasco, 2007), y los habituales sentimientos de pérdida de control derivados de la naturaleza degenerativa del trastorno (Abeles, 1991).

Teniendo en cuenta esta gran cantidad de factores intervinientes, la calidad de vida emerge como un constructo complejo, que va más allá del mero estado de salud objetivo, y se hace asimilable a la experiencia de bienestar (Schwartzmann, 2003; Velarde-Jurado y Ávila-Figueroa, 2002), conceptualizada por Diener (1984) y años más tarde por Mehlsen, Kirkegaard-Thomsen, Viidik, Olesen y Zachariae (2005), como un juicio cognitivo sobre la propia vida que puede verse afectado por el estado emocional del sujeto. De esta manera, dada la importancia que juegan en éste ámbito, tanto las variables cognitivas como las emocionales; así como la elevada prevalencia de alteraciones en estas dos esferas en población mayor y especialmente con EA, surge la necesidad de situarlas como principal foco de análisis entre los factores que guían la autopercepción de la satisfacción vital del individuo. Sin embargo, la literatura consultada acerca de la relación que establecen estos factores con la calidad de vida, arroja resultados contradictorios al respecto, ya que existen estudios que informan de una reducción de la calidad de vida con la sintomatología depresiva (Bain et al., 2003; Lucas Carrasco, 2010), así como a medida que avanza el deterioro cognitivo y funcional asociado a la demencia (Banerjee et al., 2006; Baquero, 2009; Bostrom, Jönsson, Minthon y Londos, 2007; Smith et al. 2005); mientras que otras publicaciones indican la ausencia de correlación entre el declive cognitivo y dicho constructo (Missotten et al., 2007; Schiffczyk, Romero, Jonas, Riepe, 2010; Selwood, Thorgrimsen, Orrell, 2005).

Por otra parte, existen pocos estudios que se hayan propuesto identificar la influencia que ejerce el factor de la reserva cognitiva (RC) en la calidad de vida de las personas con Alzheimer.

Desde una perspectiva comportamental, la RC podría entenderse como la capacidad del cerebro para hacer frente al daño cerebral generado por la patología, mediante procesos cognitivos preexistentes o compensatorios (Stern, 2002), desarrollados a partir de cierto nivel de actividad mental que la persona ha ido llevando a cabo a lo largo de su vida, lo que le permite retrasar la manifestación clínica de los síntomas demenciales (Valenzuela, 2008). Relacionando este constructo con la autopercepción que los pacientes manifiestan acerca de su calidad de vida, Inouye y Pedrazzani (2007), llevaron a cabo un estudio exploratorio, encontrando que los niveles más altos de escolaridad y la participación frecuente en actividades físicas, se asociaban a una autovaloración más elevada del bienestar personal de la población mayor. No obstante, otras investigaciones con objetivos similares pero en las que se empleó únicamente muestras de sujetos con bajo nivel educacional, hallaron que la valoración de dicha $\mathrm{CV}$, resultaba también elevada en cerca de la mitad de los casos estudiados (Zea Herrera et al., 2008).

Debido a la escasez de datos y a los resultados confusos acerca de varios de los aspectos mencionados de la CV y sus factores influyentes, nos proponemos llevar a cabo un estudio en el que se plantean los siguientes objetivos: (1) Examinar la auto-percepción de la calidad de vida (en sus distintas dimensiones) en el envejecimiento patológico (EA), teniendo en cuenta la RC de los individuos; y (2) Analizar la relación que tanto la sintomatología depresiva como el estado cognitivo mantienen con la CV en función de la RC de pacientes con EA.

\section{Método}

\section{Participantes}

La muestra utilizada en la investigación estaba compuesta por 112 sujetos que participaron voluntariamente en el estudio y que se distribuyeron en dos grupos: uno de 74 sujetos formado por sujetos diagnosticados de Enfermedad de Alzheimer esporádica de inicio tardío (EA) de leve a moderada, y otro de 38 sujetos que en el momento de la exploración no presentaban ningún tipo de trastorno neurológico ni psiquiátrico, ni tampoco historia de alcoholismo o toxicomanía.

Los sujetos con EA fueron seleccionados en el Servicio de Neurología del Hospital Clínico Universitario de Salamanca. La selección de los sujetos que forman el grupo control se inició cuando ya estaba avanzada la del grupo clínico, con el fin de equipararlos en edad, nivel socioeconómico y nivel educativo. Los sujetos incluidos en este grupo fueron reclutados entre los acompañantes de los pacientes que acudían a este Servicio.

Con respecto al grupo clínico, todos presentaban dominancia manual diestra. El 78\% tenían un nivel de estudios primarios, el $6 \%$ secundarios y el $16 \%$ restante, superiores. En cuanto a la situación laboral y, teniendo en cuenta la edad de la muestra, los sujetos que habían tenido con anterioridad una actividad laboral en el momento de la exploración, eran pensionistas. El nivel socioeconómico es bajo para el $8 \%$, medio para el $76 \%$ y alto para el $16 \%$.

La edad media al inicio de los síntomas fue de 71.66 años, con un mínimo de 60 años y un máximo de 86 años, de los cuales 35 pertenecían al sexo femenino y el resto al masculino.

El tiempo medio de evolución de la enfermedad fue de 2.4 años con un mínimo de un mes y un máximo de 6 años.

Referente al grupo control, también todos presentaban dominancia manual diestra. El 2\% sabía solamente leer y escribir, el $68 \%$ tenían un nivel de estudios primarios, el 13\% medios y el 17\% restante tenía estudios superiores. La situación laboral era similar a la que presentaba el grupo clínico. 
El nivel socioeconómico es bajo para el 2\%, medio para el $91 \%$ y alto para el 7\% restante. La edad media de los sujetos de este grupo, era de 70.52 años con un mínimo de 60 años y un máximo de 83 , de los cuales 20 pertenecían al sexo femenino y el resto al masculino.

En las variables anteriormente citadas (nivel educacional y edad), no se apreciaron diferencias estadísticamente significativas entre los sujetos de ambos grupos.

\section{Instrumentos}

La valoración de cada sujeto del grupo clínico comprendía una exploración neurológica completa realizada en el Servicio de Neurología del Hospital Clínico de Salamanca y una entrevista semiestructurada sobre aspectos sociodemográficos y clínicos.

Para la valoración de la gravedad de la demencia, se utilizó el Mini Mental State Examination (MMSE; Folstein, Folstein y Mchugh, 1975), adaptación de Lobo (Lobo, Ezquerra, Bugarda, Sala y Seva, 1979), considerándose los siguientes puntos de corte: Función cognitiva normal 27-30, Déficit cognitivo leve 21-26 y Déficit cognitivo importante 11-20.

Para valorar esta autoconciencia de los déficits de forma adecuada, los profesionales requieren del uso de potentes autoinformes que aporten información sobre múltiples áreas. En este sentido, emerge el Inventario de Depresión de Beck (BDI; Beck, Rial y Rickels, 1974), como el instrumento más popular para valorar la autopercepción de sintomatología depresiva, a pesar de que su estructura factorial no está del todo clara dado que las agrupaciones de información reportadas parecen variar en función de las características de la muestra. Bentz y Hall (2008), llevaron a cabo un análisis factorial de esta prueba con una muestra de pacientes mayores con algún diagnostico de demencia, depresión o ambos, hallando tres componentes principales: autoimagen negativa, anhedonia y síntomas vegetativos. Sin embargo, no siempre se ha encontrado una replicación de esta estructura al utilizar otras muestras, por lo tanto no es fácil conocer si la autoconciencia afecta por igual a todos los dominios. La fiabilidad obtenida para los ítems del Inventario de Depresión en nuestra muestra ha sido de .89 . Al igual que en la valoración de la gravedad de la demencia, también se han categorizado las puntuaciones en tres grupos, siguiendo los puntos de corte habitualmente aceptados (Beck, Steer y Garbin, 1988) para graduar la intensidad sintomatología anímica: 0-9 (ausencia); 10-18 (leve) y superior a 18 (moderado-severo).

Por otra parte, en el presente estudio se ha utilizado el cuestionario de salud SF-36 para evaluar la autopercepción de la calidad de vida de la muestra. Según Vilagut et al. (2008), es uno de los instrumentos generales más empleados para evaluar la calidad de vida relacionada con la salud tanto en personas con alguna patología, como en la población general. Consta de 35 ítems clasificados en 8 dimensiones: función física, rol físico, dolor corporal, salud general, vitalidad, función social, rol emocional, y salud mental. Asimismo, existe un ítem adicional que no pertenece a las dimen- siones anteriores y que evalúa el cambio en la salud con el tiempo. Dicho ítem de transición pregunta sobre la variación en el estado de salud general respecto al año anterior (Vilagut et al., 2005). En cada dimensión del SF-36, los ítems se codifican siguiendo un gradiente ascendente hasta las mayores puntuaciones que implican un mejor estado de salud, a continuación, se suman las puntuaciones y se transforman en una escala con rango de 0 (peor estado de salud) hasta 100 (mejor estado de salud). Estas modificaciones se han realizado siguiendo las indicaciones que ofrece el manual de puntación e interpretación del cuestionario (Ware y Sherbourne 1992). La prueba fue cumplimentada por los pacientes ayudados por los cuidadores, pero sin que estos aportaran datos.

Las tasas de no-respuesta a los ítems del SF-36 fueron consistentemente bajas e inferiores al $1 \%$ tanto en el caso del grupo control como en el grupo clínico. La fiabilidad alcanzada por todas las dimensiones fue superior a .70 en ambos grupos. En el caso del grupo control, el valor mínimo se encontró en la dimensión de Función social (.70) mientras que la mayor puntuación se registró en la dimensión del Rol físico (.96). En el caso del grupo clínico el valor más bajo se situó en las dimensiones Salud general y Vitalidad (ambas con un alfa de .76) y el valor máximo aparece en la dimensión Rol físico con .90 .

En relación a la RC, la teoría establece que una serie de factores, congénitos o ambientales, proporcionan mecanismos cuantitativos y cualitativos que hacen al sujeto más resistente a los procesos patológicos cerebrales. El individuo con mayor reserva podría soportar más patología antes de que aparecieran las manifestaciones clínicas, que aquel otro con una menor reserva. Sánchez, Torrellas, Martín y Barrera (2011) proponen un modelo para clasificar a los pacientes en alta y baja reserva cognitiva, en base a las puntuaciones que toman de las variables socioeconómicas de los pacientes. Los factores que intervienen en el modelo son: Escolarización del padre (ns/nc, estudios no superiores, estudios superiores); Cualificación de la profesión del padre (ns/nc; no cualificadocualificado; altamente cualificado); Estudios del paciente (estudios no superiores; estudios superiores); Ocupación del paciente (no cualificado-cualificado; altamente cualificado); Implicación cognitiva de dichas actividades lúdicas (ns/nc, No Si); Compromiso social (ns/nc; no o baja; moderada-alta) Actividad Intelectual (ns/nc; no o baja; moderada-alta); Lectura actual (ns/nc no-bajamoderada, alta). La información en relación a estas variables se ha recogido mediante la entrevista semiestructurada mencionada anteriormente, y en función de las puntaciones obtenidas en ellas, se han clasificado a los sujetos en alta y baja reserva cognitiva.

\section{Procedimiento}

Todos los participantes firmaron la hoja de consentimiento informado.

Para la inclusión de los sujetos en el grupo clínico, debían satisfacer los siguientes criterios: 1) Diagnóstico neurológico y neuropsicológico de EA de acuerdo con los criterios 
del DSM-IV (DSM-IV, 1995); 2) Edad de corte de 60 años para diferenciar entre inicio temprano y tardío según parámetros establecidos por los investigadores y con base en las observaciones de la presentación precoz y tardía en la población estudiada por el grupo. 3) Los criterios del grupo de trabajo NINCDS-ADRDA para el diagnóstico de EA probable (Mckhann et al., 1984).

Para los sujetos con EA se utilizaron pruebas diagnósticas para la exclusión de aquellos individuos con posible demencia secundaria. Dentro de estas pruebas se incluyeron: función tiroidea, serología luética, niveles de vitamina B12 y ácido fólico.

Todos los sujetos con EA se sometieron a un examen neurorradiológico mediante TAC. La totalidad de los sujetos incluidos en la muestra clínica, presentaban, según los informes neurorradiológicos, lesiones estructurales consistentes en la presencia de distintos grados de atrofia cerebral preferentemente cortical. Se descartaron del estudio aquellos sujetos que presentaban o habían presentado trastornos psiquiátricos. Asimismo, se excluyeron los sujetos con defectos motores o sensoriales, excepto los visuales corregidos con lentes.

\section{Análisis estadísticos}

Para cada dimensión de salud se ha calculado la media y la desviación típica para los individuos de alta reserva cogni- tiva y de baja reserva, tanto en el grupo de enfermos de Alzheimer como en el grupo control. Se ha utilizado el alfa de Cronbach con el objetivo de conocer la fiabilidad de cada dimensión. Para comparar en cada escala las diferencias entre los grupos de alta y baja reserva cognitiva considerando el grupo control y el grupo de Alzheimer, se ha utilizado el análisis de la varianza de dos factores independientes (ANOVA II). Al detectar interacción entre los factores se ha utilizado el t-test para analizar las diferencias en cada uno de los grupos por separado, previamente comprobando la homogeneidad de las varianzas con la prueba de Levene. Además, se ha calculado la correlación de Spearman para conocer la relación de las dimensiones de salud con el funcionamiento cognitivo y sintomatología depresiva.

\section{Resultados}

En la Tabla 1, se reflejan las características demográficas de la muestra utilizada, así como los resultados globales obtenidos en la escala cognitiva (MMSE) y en la valoración del estado de ánimo (BDI), tanto en el grupo clínico como en el grupo control, divididos en función de su reserva cognitiva.

Como podemos observar no existen diferencias significativas en la edad entre los distintos grupos.

Tabla 1. Características sociodemográficas de la muestra utilizada, resultados globales del MMSE y BDI.

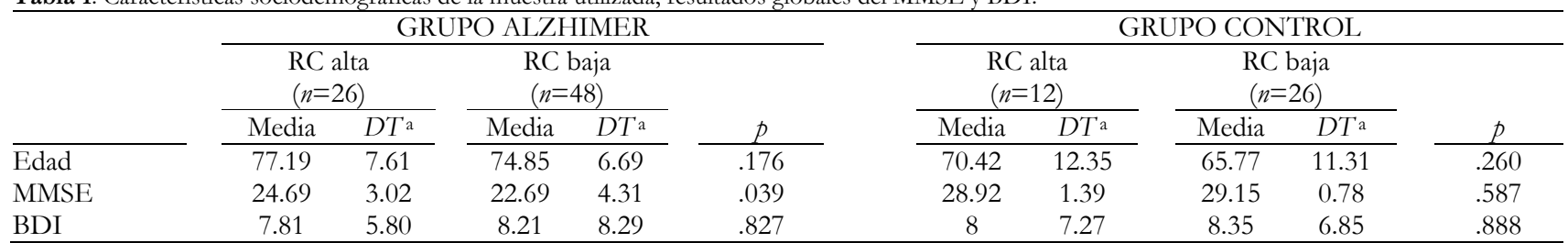

a DT: desviación típica.

Con respecto al estado cognitivo, encontramos una puntuación promedio más elevada en los pacientes con RC alta, en relación a los sujetos con baja reserva, siendo significativa la diferencia. Por el contrario, en el grupo control todos los participantes se situaron en el rango correspondiente al funcionamiento cognitivo normal (MMSE >27).
En relación con la variable estado de ánimo, el BDI informó de un comportamiento similar en los sujetos incluidos en ambas categorías de RC, tanto en el grupo con patología como en el grupo control.

Los resultados del cuestionario de autovaloración de la calidad de vida SF-36 se exponen en la Tabla 2.

Tabla 2. Descriptivos y significación obtenidas en los grupos de alta y baja reserva cognitiva para cada una de las dimensiones de la escala de autovaloración SF-36 aplicadas a los grupos de EA y Control.

\begin{tabular}{|c|c|c|c|c|c|c|c|c|c|c|c|c|c|c|}
\hline \multirow{3}{*}{$\begin{array}{l}\text { Subescalas de } \\
\text { SF-36 }\end{array}$} & \multicolumn{7}{|c|}{ GRUPO ALZHEIMER } & \multicolumn{7}{|c|}{ GRUPO CONTROL } \\
\hline & \multicolumn{3}{|c|}{ RC alta } & \multicolumn{3}{|c|}{ RC baja } & \multirow[b]{2}{*}{$p$} & \multicolumn{3}{|c|}{$\mathrm{RC}$ alta } & \multicolumn{3}{|c|}{ RC baja } & \multirow[b]{2}{*}{$p$} \\
\hline & $\mathrm{n}$ & Media & $D T^{\mathrm{a}}$ & $\mathrm{N}$ & Media & $D T^{\mathrm{a}}$ & & $\mathrm{n}$ & Media & $D T^{\mathrm{a}}$ & $\mathrm{n}$ & Media & $D T^{\mathrm{a}}$ & \\
\hline$\overline{\text { Salud General }}$ & 24 & 63.67 & 14.49 & 46 & 60.78 & 13.74 & .41 & 11 & 65.82 & 15.22 & 26 & 60.77 & 14.36 & .34 \\
\hline Función Física & 25 & 78.93 & 17.47 & 46 & 74.49 & 17.51 & .31 & 11 & 85.76 & 14.69 & 26 & 83.33 & 16.87 & .68 \\
\hline Rol físico & 25 & 92.5 & 16.14 & 46 & 81.79 & 21.52 & .02 & 11 & 79.55 & 23.90 & 26 & 87.02 & 21.06 & .35 \\
\hline Rol emocional & 25 & 88 & 18.33 & 44 & 78.41 & 23.73 & .066 & 11 & 81.82 & 22.92 & 26 & 79.49 & 24.18 & .79 \\
\hline Función Social & 24 & 85.98 & 15.16 & 45 & 73.54 & 22 & .008 & 11 & 75.21 & 24.76 & 26 & 82.87 & 22.17 & .36 \\
\hline Dolor corporal & 24 & 74.24 & 23.32 & 46 & 70.55 & 20.84 & .50 & 11 & 76.03 & 18.31 & 26 & 72.38 & 20.49 & .61 \\
\hline Vitalidad & 24 & 69.44 & 20.58 & 46 & 64.22 & 15.23 & .23 & 11 & 68.18 & 20.69 & 26 & 62.66 & 16.81 & .40 \\
\hline Salud Mental & 24 & 76.25 & 17.89 & 46 & 65.58 & 15.95 & .018 & 11 & 73.33 & 16.67 & 26 & 71.41 & 16.09 & .74 \\
\hline
\end{tabular}

a DT: desviación típica. 
Se han analizado de manera conjunta las puntuaciones obtenidas por los cuatro subgrupos del estudio, mediante un ANOVA de dos factores con interacción, obteniéndose una interacción significativa únicamente en las dimensiones Función social $(F(1,102)=4.74, p=.032)$ y Rol físico $(F$

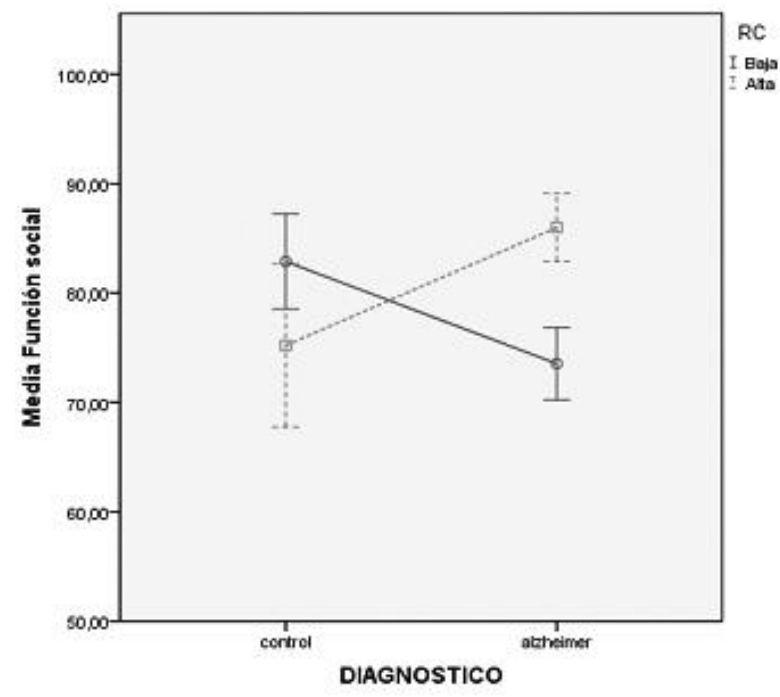

A
$(1,102)=4.1, p=.032)$. Esto implica que existe una influencia combinada de las variables RC y EA en ambas facetas y podría ser responsable de la modificación de la tendencia descrita y de la magnitud cuando se da una ausencia de dicha patología (Figura 1).

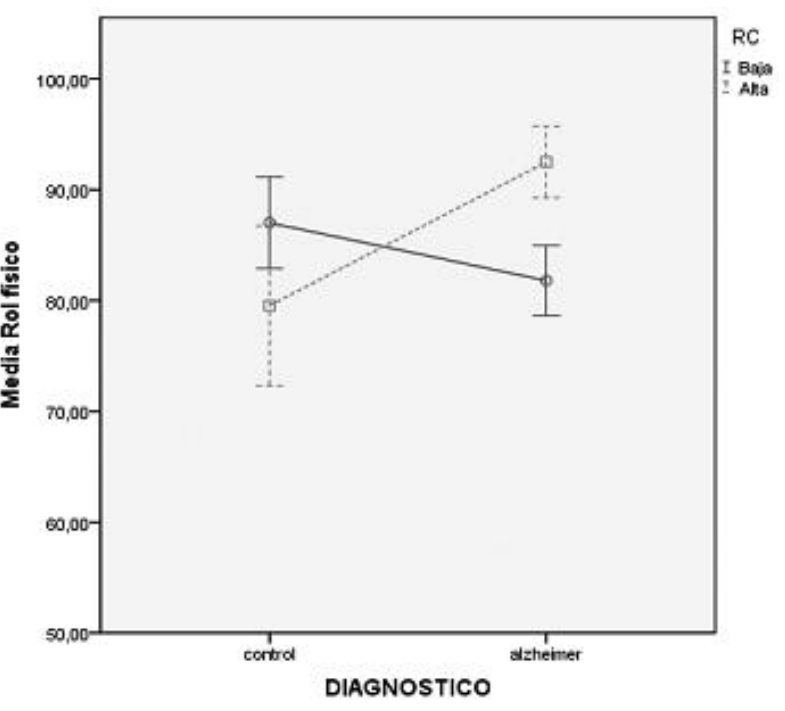

B

Figura 1. Media \pm Error estándar para el Rol físico (A) y Función Social (B) para los grupos control y clínico en función de la reserva cognitiva.

Cuando se realiza el t-test entre el grupo de baja y alta reserva cognitiva para el grupo control y el grupo clínico por separado, se detectan diferencias significativas en el caso de la Función social y en la dimensión de Rol físico para el grupo con patología.

En el caso de la dimensión Salud Mental aunque la interacción no ha sido estadísticamente significativa ( $F$ $(1,103)=1.62, p=.20)$, se observa un tendencia diferencial entre el grupo control y clínico (Figura 2).

Si se realiza el contraste t-test en el grupo con patología se detectan diferencias significativas entre los grupos de alta y baja reserva cognitiva.

Dado que la autopercepción del estado de salud podía verse influida por algunos factores como el estado mental o la sintomatología depresiva, se calcularon las posibles correlaciones de estas variables con las escalas del cuestionario. En el caso del funcionamiento cognitivo, los valores de correlación de Spearman fueron estadísticamente no significativos con respecto a todas las escalas de salud y en ambos grupos experimentales. En el grupo con patología, la mayor magnitud se obtuvo con Salud mental $\left(r_{s}=.14\right)$ y en el grupo control con Rol emocional y Dolor corporal $\left(r_{s}=-.27\right.$, en ambos). Por otro lado, se han encontrado correlaciones inversas significativas entre las variables autopercepción de dicha CV y la sintomatología depresiva. De este modo, los participantes que refirieron un mayor número de síntomas depresivos, evaluaron su calidad de vida con puntuaciones significativamente más bajas. La magnitud de las correlaciones es mayor en el grupo control variando entre el menor valor $r_{s}=-.43$ para Dolor corporal y la mayor magnitud para Vitalidad $\left(r_{s}=-.70\right)$. En el grupo clínico las magnitudes de correlación varían entre $r_{s}=-.32$ para Función Social y $r_{s}=-.47$ para Vitalidad y Salud Mental.

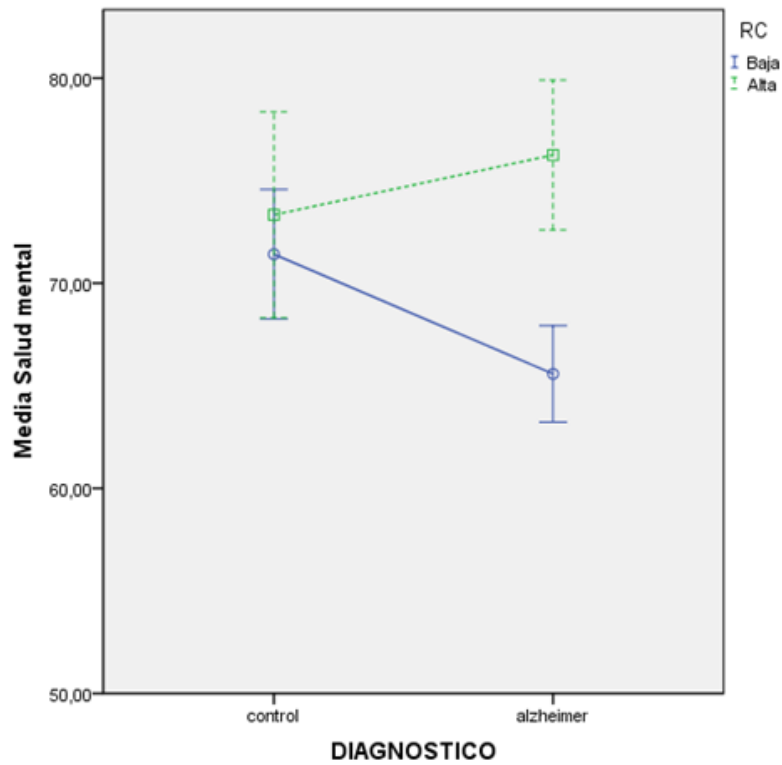

Figura 2. Valores medios de Salud mental \pm Error estándar para el grupo control y clínico en función de la reserva cognitiva.

Posteriormente, con la intención de analizar el papel que juega la RC en las relaciones que la cognición y el estado de ánimo mantienen con la percepción de la $\mathrm{CV}$, realizamos 
una nueva categorización de las puntuaciones obtenidas en la muestra con demencia. De este modo, distribuimos los valores de los sujetos con alta y baja RC en tres niveles representativos del grado de deterioro en cada una de las variables mencionadas, quedando recogidos estos datos en la Tabla 3. El nivel de déficit cognitivo moderado no se ha contemplado porque el tamaño muestral en el grupo de RC alta era sólo de dos individuos.

Tabla 3. Puntuaciones medias y significación obtenidas por el grupo EA en las subescalas de la prueba SF-36 según su funcionamiento cognitivo.

\begin{tabular}{|c|c|c|c|c|c|c|c|c|c|c|c|c|c|c|c|c|c|c|c|}
\hline \multirow{3}{*}{$\begin{array}{l}\text { Subescalas } \\
\text { de SF-36 }\end{array}$} & \multicolumn{7}{|c|}{$\begin{array}{l}\text { Funcionamiento Cognitivo Normal } \\
\text { (MMSE 27-30) }\end{array}$} & \multicolumn{7}{|c|}{$\begin{array}{l}\text { Déficit Cognitivo Leve } \\
\text { (MMSE 21-26) }\end{array}$} & \multicolumn{5}{|c|}{$\begin{array}{l}\text { Déficit Cognitivo Moderado } \\
(\text { MMSE 11-20) }\end{array}$} \\
\hline & \multicolumn{3}{|c|}{ RC Alta } & \multicolumn{3}{|c|}{ RC Baja } & \multirow[b]{2}{*}{$p$} & \multicolumn{4}{|c|}{ RC Alta } & \multicolumn{3}{|c|}{ RC Baja } & \multicolumn{2}{|c|}{ RC Alta } & \multicolumn{2}{|c|}{ RC Baja } & \multirow[b]{2}{*}{$n$} \\
\hline & $\overline{M^{\mathrm{a}}}$ & $D T^{\text {ь }}$ & $n$ & $M^{\text {a }}$ & DT в & $n$ & & $M^{\text {a }}$ & $D T^{\text {в }}$ & $n$ & $M^{\text {a }}$ & $D T^{\mathrm{b}}$ & $n$ & $p$ & $M^{\text {a }}$ & $D T^{\text {в }}$ & $\mathrm{n} \overline{M^{\mathrm{a}}}$ & $D T^{\text {b }}$ & \\
\hline . & 65.2 & 12.23 & 10 & 62.18 & 10.64 & 11 & .55 & 62.33 & 16.92 & 12 & 59.79 & 16.94 & 19 & .687 & 64 & 16.97 & $2 \longdiv { 6 1 }$ & 12 & 16 \\
\hline un & 73 & 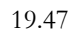 & 10 & 76.67 & 13.08 & 11 & .615 & 84.87 & 15.61 & 13 & 72.46 & 17.84 & 19 & .051 & 70 & & 275.42 & 20 & 16 \\
\hline $\mathrm{CO}$ & 1.5 & & 10 & 85.23 & 19.22 & 11 & .102 & 95.19 & 15.76 & 13 & 78.29 & 22.38 & 19 & .018 & 100 & . & 283.59 & 22.69 & 16 \\
\hline & 93.33 & 161 & 10 & 71.21 & 24.82 & 11 & .026 & 83.33 & 20.41 & 13 & 79.63 & 25.92 & 18 & & 91.6 & & 282.22 & 20.38 & 15 \\
\hline . & 4.55 & & 10 & 79.34 & 17.76 & & & 84.85 & 14.15 & 12 & 72.73 & 24.62 & 19 & .133 & 100 & 0. & 270.30 & 21.86 & 15 \\
\hline Dolo & 74.55 & 23.78 & 10 & 71.9 & 23.52 & 11 & .801 & 73.48 & 25,26 & 12 & 69.86 & 22.28 & 19 & .678 & 77.27 & 19.28 & 270.45 & 18.33 & 16 \\
\hline Vitalidad & 70.42 & 20.55 & 10 & 64.39 & 15.85 & 11 & .459 & 68.06 & 22.98 & 12 & 61.84 & 17.47 & 19 & .400 & 72.92 & 8.84 & 266.93 & 12.12 & 16 \\
\hline Salud Mental & 74.57 & 21.15 & 10 & 68.79 & 18.52 & 11 & .505 & 77.22 & 17.16 & 12 & 63.33 & 17.14 & 19 & .036 & 78.33 & 7.07 & 266.04 & 12.95 & 16 \\
\hline
\end{tabular}

a M: Media aritmética; ${ }^{b}$ DT.: desviación típica. Nota: cuando los tamaños muestrales son pequeños no se calcula el valor de $p$.

Centrándonos en un análisis más detallado de los mismos, y considerando en primer lugar el estado cognitivo, podemos observar que los pacientes con mayor RC del subgrupo con ausencia de deterioro, presentaron medias superiores en todas las dimensiones excepto en la Función física, sin embargo, de todas ellas, las únicas puntuaciones que arrojaron diferencias significativas con respecto a los participantes con menor RC, fueron las correspondientes al Rol emocional.
En relación a los participantes con deterioro cognitivo leve, volvemos a observar el patrón consistente en una mejor autopercepción de la CV en los individuos con RC superior, pero en este caso la significación sólo se alcanzó en las facetas Rol físico y Salud mental.

En segundo lugar, si atendemos a los valores correspondientes a los tres niveles formados en relación a la sintomatología depresiva en el grupo clínico se observan algunas diferencias con respecto a los resultados anteriores (Tabla 4).

Tabla 4. Puntuaciones medias y significación obtenidas por el grupo EA en las subescalas de la prueba SF-36 según su sintomatología depresiva.

\begin{tabular}{|c|c|c|c|c|c|c|c|c|c|c|c|c|c|c|c|c|c|c|c|}
\hline \multicolumn{20}{|c|}{ SINTOMATOLOGÍA DEPRESIVA } \\
\hline \multirow{3}{*}{$\begin{array}{l}\text { Subescalas } \\
\text { de SF-36 }\end{array}$} & \multicolumn{6}{|c|}{$\begin{array}{l}\text { Ausencia síntomas } \\
\text { (BDI 0-9) }\end{array}$} & \multicolumn{7}{|c|}{$\begin{array}{l}\text { Sintomatología Leve } \\
\text { (BDI 10-18) }\end{array}$} & \multicolumn{6}{|c|}{$\begin{array}{l}\text { Sintomatología moderada-grave } \\
(\text { BDI }>18)\end{array}$} \\
\hline & $\overline{\mathrm{RC} \mathrm{Al}}$ & & & $\mathrm{RC} \mathrm{Ba}$ & & & $\overline{\mathrm{RC} \mathrm{Al}}$ & & & $\overline{\mathrm{RC} \mathrm{Ba}}$ & & & & RC Alta & & & RC Baja & & \\
\hline & $M^{\text {a }}$ & $D T^{\mathrm{b}}$ & $\bar{n}$ & $M^{\text {a }}$ & $D T^{\mathrm{b}}$ & $p$ & $M^{\mathrm{a}}$ & $D T^{\mathrm{b}}$ & $\bar{n}$ & $M^{\text {a }}$ & $D T^{\mathrm{b}}$ & $n$ & $p$ & $M^{\text {a }}$ & $D T^{\mathrm{b}}$ & $n$ & $M^{\text {a }}$ & $D T^{\mathrm{b}}$ & $n$ \\
\hline Salud General & 66.40 & 14.09 & 15 & 64.77 & 13.20 & 31.703 & 59.50 & 15.78 & 8 & 1.64 & 9.37 & 11 & .191 & 56.00 & & 1 & 55.00 & 16.77 & 4 \\
\hline Función Física & 78.75 & 17.46 & 16 & 80.00 & 15.59 & 31.804 & 77.92 & 19.35 & 8 & 64.55 & 17.66 & 11 & .136 & 90.00 & & 1 & 59.17 & 11.98 & 4 \\
\hline Rol físico & 96.09 & 9.92 & 16 & 88.31 & 17.06 & 31.100 & 87.50 & 24.09 & 8 & 75.00 & 25.00 & 11 & .290 & 75.00 & & 1 & 50.00 & .00 & 4 \\
\hline Rol emocional & 96.87 & 6.72 & 16 & 79.31 & 23.00 & 29.001 & 75.00 & 21.82 & 8 & 83.33 & 25.82 & 11 & .470 & 50.00 & & 1 & 58.33 & 16.67 & 4 \\
\hline Función Social & 89.70 & 10.79 & 15 & 79.39 & 16.87 & 30.037 & 80.68 & 20.87 & 8 & 66.12 & 27.30 & 11 & .224 & 72.73 & & 1 & 50.00 & 24.05 & 4 \\
\hline Dolor corporal & 83.03 & 18.78 & 15 & 74.49 & 19.56 & 31.167 & 62.50 & 23.52 & 8 & 62.81 & 20.51 & 11 & .976 & 36.36 & & 1 & 61.36 & 28.14 & 4 \\
\hline Vitalidad & 75.83 & 16.00 & 15 & 68.68 & 15.21 & 31.149 & 59.38 & 25.37 & 8 & 53.79 & 8.22 & 11 & .565 & 54.17 & & 1 & 58.33 & 17.01 & 4 \\
\hline Salud Mental & 84.44 & 10.59 & 15 & 69.35 & 15.38 & 31.001 & 65.00 & 19.60 & 8 & 59.39 & 15.83 & 11 & .499 & 43.33 & & 1 & 53.33 & 11.22 & 4 \\
\hline
\end{tabular}

Sólo 5 pacientes obtuvieron puntuaciones superiores a 18, por lo que no se consideró el grupo de sintomatología más grave. En relación al grupo con valores más bajos de depresión, se observa como las puntaciones medias son más elevadas en el grupo de mayor RC, excepto en el Rol físico. Se detectan diferencias estadísticamente significativas en las escalas Salud mental, Rol emocional y Función social. En el caso del grupo con un estado de ánimo comprendido entre 10-18 puntos en el BDI, el comportamiento es diferente, si bien los tamaños muestrales son más pequeños. En la mayoría de las escalas de salud se presentan mayores valores medios en el grupo de RC alta, excepto en Rol emocional en el que es mayor la media en el grupo de RC baja, y en la escala Dolor corporal que presentan valores medios muy similares.

\section{Discusión}

Atendiendo al tema central de nuestra investigación, consistente en el estudio de la autopercepción que poseen las personas con EA acerca de su calidad de vida, el primer dato relevante que arrojan nuestros resultados, indica el carácter positivo con el que los mayores de ambos grupos, juzgaron su calidad de vida general, ya que los cuatro subgrupos que 
componen este estudio presentaron valores por encima de los 50 puntos en cada una de las 8 subescalas de la prueba. Estos resultados aparecen de forma reincidente en numerosas investigaciones realizadas en diferentes poblaciones: grupos de la tercera edad diagnosticados con demencia (James, Xie y Karlawish, 2005) y sin ella (Castellón y Romero, 2004; Zúniga, Carrillo, Fos, Gandek y Medina-Moreno, 1999); y grupos de sujetos con menor edad pero con riesgo de padecer EA (Zea Herrera et al., 2008). No obstante, algunos autores, proponen tomar este resultado con cautela, ya que puede verse influido por algunas variables moderadoras que podrían alterar su validez, como el excesivo optimismo con el que los sujetos con demencia evalúan su CV (Vogel et al., 2004) o la minimización de los efectos negativos de la EA, a medida que el paciente va ganando experiencia con la patología (Banerjee et al., 2006).

Por otro lado, los análisis de nuestra investigación informan que las puntuaciones máximas en el cuestionario de salud se sitúan en la dimensión rol físico en ambos grupos (con y sin patología), mientas que los valores mínimos aparecen en la faceta social, en el caso del grupo control, y las escalas salud general y vitalidad en el caso de la muestra con EA. Estos resultados, son similares a los hallados en otras publicaciones. Ramírez, Agredo, Jerez y Chapal (2008), llevaron a cabo un estudio exploratorio sobre la valoración de la $\mathrm{CV}$ en población mayor sana, encontrando que los aspectos mejor valorados eran los relativos a los dominios de vitalidad, salud mental y salud física, sin embargo, las puntuaciones más bajas correspondían al rol emocional y la función social. Asimismo, el estudio de Lucas-Carrasco et al. (2011), indicó que, entre todas las variables, el estado de salud era el que se relacionaba de forma más significativa con la calidad de vida percibida por individuos con demencia.

En relación a como la reserva cognitiva, actúa sobre valoración de la calidad de vida, los datos del presente estudio informan que los individuos con mayor RC obtuvieron puntuaciones más elevadas en el cuestionario de salud SF-36 lo cual parece convertirse en una tendencia, al ponerse de manifiesto tanto en el grupo clínico como en el grupo control. Aunque no hemos encontrado ninguna investigación que examinara la relación entre este constructo a nivel global y la $\mathrm{CV}$, si existen publicaciones orientadas a la hora de analizar de forma aislada como algunas variables pertenecientes a la $\mathrm{RC}$, se asocian a la calidad de vida percibida, arrojando conclusiones compatibles con nuestros resultados. En este sentido, destaca el estudio de Ferrando, Nebot, Borrell y Egea (1996), en el que examinando la percepción del estado de salud en población mayor no institucionalizada, se encontró que las mujeres que peor valoraban su salud eran aquellas con un bajo nivel educativo y falta de apoyo en las actividades cotidianas, sin embargo en el caso de los varones, la percepción negativa de la salud estaba más asociada a la ausencia de apoyo emocional. Resultados equivalentes aparecen en otros estudios donde se ponen de manifiesto asociaciones significativas que relacionan la autovaloración negativa de la calidad de vida con el bajo nivel educacional (Inouye y Pe- drazzani, 2007) y con las carencias en apoyo social y emocional (Azpiazu et al., 2002; Fernández-Merino, Vérez, y Gude, 1996). En esta línea, otros estudios, han demostrado como otros componentes del constructo RC, tales como el elevado nivel ocupacional logrado en la etapa laboral (Arber y Ginn, 1993; Séculi et al., 2001), la práctica de ejercicio físico (Inouye y Pedrazzani, 2007) o las relaciones sociales (Livingston et al., 2008), también correlacionan de forma directa con una calidad vital percibida de manera favorable en la tercera edad.

Asimismo, como exponíamos en el apartado de resultados, la asociación entre ambas variables (RC y CV percibida) que apareció en la muestra con patología, únicamente alcanzó la significación en las dimensiones: Rol físico, Función social y Salud Mental. De este aspecto, podemos inferir que aquellos pacientes con EA y una elevada RC, son más propensos a considerar (con respecto a pacientes con una RC inferior), que su vida está regida por una sensación de felicidad, que presentan escasos problemas cotidianos en base a su salud física, y que sus posibles alteraciones en la salud interfieren escasamente en sus actividades sociales. De este modo, parece ser que con la evolución de la patología, aparece un descenso más notable en las escalas físicas y sociales, lo que supone para aquellos individuos con una reserva más baja y un menor número y calidad de estrategias de afrontamiento, una pérdida funcional más acusada, lo que acaba traduciéndose en una valoración menos positiva de su calidad vital. Además, no podemos olvidar que la interacción entre RC y EA sólo resultó significativa en las facetas Rol físico y Función social, por lo que podrían ser consideradas como más discriminativas en la evaluación de la CV para este tipo de población. Algunos autores (Logsdon, 2002; Oswald, 2004), ya habían señalado que el aspecto físico era la variable que mejor diferenciaba a los sujetos con demencia en función de su autovaloración de la CV. Sin embargo, como ya comentábamos, otros autores encontraban que la asociación más significativa se localizaba en otras facetas como el estado de salud general (Lucas-Carrasco et al., 2011).

En cuanto al estado cognitivo de los individuos, nuestros datos señalan una ausencia de correlación entre las puntuaciones del MMSE y la calidad de vida percibida, lo cual coincide con los datos publicados en otras investigaciones (Banerjee et al., 2006; Logdson, 2002; Woods, Thorgrimsen, Spector, Royan y Orrell, 2006). Sin embargo, también hemos localizado algunos trabajos, donde la asociación entre estas dos variables resultó ser significativa e inversa (Baquero, 2009; Sloane et al., 2005). Nagamoto, Kita, Takigawa, Nomaguchi y Sameshima (1997) propusieron que los déficit en el funcionamiento cognitivo podían afectar de manera indirecta a la percepción de la calidad de vida del paciente con demencia, siendo responsables de las alteraciones conductuales que reducían parte de la capacidad funcional del sujeto. Esta hipótesis podría servir para explicar la confusión que existe en la bibliografía en relación a este aspecto. De hecho, en esta misma dirección, Baquero (2009) postuló que 
los aspectos cognitivos no aportan información adicional en relación con la calidad de vida, más allá de la contribución conseguida con la evaluación de los aspectos funcionales. Asimismo, en relación a la explicación de este aspecto contradictorio, sugerimos como una hipótesis que requiere una investigación futura más profunda, que la RC también podría influir en la relación entre estado cognitivo y la calidad de vida percibida, dado que al analizar diferentes grados de deterioro cognitivo en sujetos categorizados en función de su reserva, hemos detectado correlaciones significativas en determinados dominios del cuestionario de salud SF-36.

Al contrario de lo que ocurría con el estado mental, la sintomatología depresiva sí mostró una gran asociación con la percepción de la calidad de vida tanto en sujetos con EA como en el grupo control. Este mismo resultado aparece de forma reiterada en otros trabajos (Azpiazu et al. 2002; Lapid et al., 2011; Logsdon, Gibbons, McCurry y Teri, 1999), lo que ha llevado a afirmar a autores como Thorgrimsen et al. (2003) que el estado de ánimo es el principal determinante de la calidad de vida en todas las etapas de la demencia. En este sentido, Hoe et al. (2009) llevaron a cabo un estudio con 238 pacientes con demencia, en el que valoraron a lo largo de 20 semanas, la percepción de la $\mathrm{CV}$ de los propios pacientes y sus cuidadores, concluyendo que el descenso en la calidad de vida de los pacientes, se podría predecir mediante la línea base de depresión y ansiedad, la calidad de vida previa y el deterioro cognitivo conseguido con el progreso de la enfermedad (Hoe et al. 2009). No obstante, la mayor parte de las publicaciones coinciden en afirmar que el estado emocional juega un papel más relevante en la calidad de vida percibida, que la gravedad de la demencia (Banerjee et al., 2006; Lapid et al., 2011).

Teniendo en cuenta lo expuesto hasta el momento, debemos señalar como la evidencia empírica, pone de manifiesto la importancia de realizar valoraciones frecuentes de la calidad de vida en personas con demencia, como vía para optimizar los tratamientos psicosociales que permitan minimizar el impacto de la enfermedad y su curso. En este sentido, llevar a cabo intervenciones con el objetivo de producir una mejora del funcionamiento cognitivo (Hertzog, Kramer, Wilson y Lindenberger, 2008), reducir el malestar emocional (Hoe et al., 2009), o favorecer el incremento de la RC, podrían ayudar al paciente a considerar su calidad de vida con un carácter más positivo, reflejándose en un afrontamiento de la enfermedad más favorable.

No obstante, debemos mencionar algunas limitaciones de nuestro estudio que pueden servir como punto de partida para el planteamiento de nuevas hipótesis en la presente línea de investigación. Una de las restricciones del estudio radica en el procedimiento de selección de la muestra, tratándose de un método no probabilístico en el que los participantes no fueron elegidos de forma aleatoria. Este tipo de muestreo reduce en cierto grado la posibilidad de generalización de los resultados, sin embargo es necesario matizar que los criterios de inclusión y exclusión de participantes, fueron definidos y seguidos de forma rigurosa, lo que supone una mayor aproximación a la representatividad de la muestra. En esta línea, cabe mencionar una nueva limitación del estudio, relacionada con la existencia de diferentes tamaños en los subgrupos al analizar la influencia de los factores seleccionados sobre la variable dependiente, quedando alguna de las submuestras con tal escasa representación, que no alcanzó el suficiente poder para detectar las diferencias buscadas. Otra de las limitaciones se sitúa en el hecho de haber basado las evaluaciones únicamente en la percepción del propio sujeto acerca de ese estado subjetivo que conforma la calidad de vida. Sin embargo, existen contradicciones en las diversas publicaciones sobre si los pacientes con EA conservan la capacidad para hacer valoraciones fiables en relación sus propias experiencias. Así, algunos autores han demostrado que el deterioro cognitivo no altera dicha función (Hoe, Katona, Orrell y Livingston, 2007; Schiffczyk et al., 2010; Waldorff, Nielsen y Waldemar, 2010), sin embargo, otros estudios parecen confirmar que los déficit propios del avance de la enfermedad introducirían sesgos en los resultados (Ready, Ott y Grace, 2006; Selai, Trimble, Rossor y Harvey, 2001). Es por ello, que en la línea de lo planteado por Zea Herrera et al. (2010), sugerimos que la valoración debe ser lo más integradora posible, contrastando la información con el entorno del paciente, con vistas a la obtención de una visión más global de la situación, reduciendo así las fuentes de error provenientes de cada uno de los agentes informantes. Finalmente, por su relevancia y su mayor acercamiento al constructo RC, se eligieron sólo algunas variables para estudiar su efecto en la autopercepción de la calidad de vida; sin embargo, existen otros factores como la ansiedad, el insomnio o las enfermedades crónicas que cursan con dolor, que también son susceptibles de incidir en dicha valoración subjetiva del propio bienestar. En este sentido, sería interesante que futuras líneas de investigación, se centraran en explorar los efectos de un mayor número de variables, utilizando además diferentes formatos de valoración de la calidad de vida y procedimientos aleatorios de selección de muestra.

\section{Conclusiones}

Como conclusión final, comentar que el presente estudio sugiere que la reserva cognitiva podría ser una fuente de influencia en la percepción de la calidad de vida de las personas con EA, en la medida en que sus diversos componentes conducirían a la consecución de una capacidad funcional más óptima y una aceptación del estado cognitivo y emocional más favorable. 


\section{Referencias}

Abeles, R. P. (1991). Sense of control, quality of life and frail older people. En J. Birren., J. Lubben., J. Rowe et al., (Eds.), The concept and measurement of quality of life in the frail elderly (pp. 297-314). San Diego, California: Academic Press.

Almkvist, O. \& Winblad, B. (1999). Early diagnosis of Alzheimer dementia based on clinical and biological factors. European Archives of Psychiatry and Clinical Neuroscience, 249, 3-9.

Arber, S. \& Ginn, J. (1993). Gender and inequalities in health in later life. Social Science \& Medicine, 36, 33-46. doi: 10.1016/0277-9536(93)90303-L

Azpiazu, M., Cruz, A., Villagrasa, J. R, Abanades, J. C., García-Marín, N. y Alvear, F. (2002). Factores asociados a mal estado de salud percibido o a mala calidad de vida en personas mayores de 65 años. Revista Española de Salud Pública, 76, 683-699.

Bain, G. H., Lemmon, H., Teunisse, S., Starr, J. M., Fox, H. C., Deary, I.J. \& Whalley, L. J. (2003). Quality of life in healthy old age: relationships with childhood IQ, minor psychological symptoms and optimismo. Social Psychiatry and Psychiatric Epidemiology, 38, 632-636.

Banerjee, S., Smith, S. C., Lamping, D. L., Harwood, R. H., Foley, B., Smith, P., et al. (2006). Quality of life in dementia: more than just cognition. An analysis of associations with quality of life in dementia. Journal of Neurology, Neurosurgery, and Psychiatry, 77,146-148. doi: 10.1136/jnnp.2005.072983

Baquero, M. (2009). Calidad de vida en la enfermedad de Alzheimer. Revista Neurología, 49 (7), 337-34.

Beck, A. T., Rial, W. Y. \& Rickels, K. (1974). Short form of depression inventory (cross-validation). Psychological Reports, 34, 1184-1186.

Beck A. T., Steer, R. A. \& Garbin, M. G. (1988) Psychometric properties of the Beck Depression Inventory. Twenty-five years of evaluation. Clinical Psychology Review, 8, 77-100.

Bentz, B. G. \& Hall, J. R. (2008). Assessment of depression in a geriatric inpatient cohort: A comparison of the BDI and GDS. International Journal of Clinical and Health Psychology, 8, 93-104.

Bostrom, F., Jönsson, L., Minthon, L. \& Londos, E. (2007). Patients with dementia with Lewy bodies have more impaired quality of life than patients with Alzheimer disease. Alžbeimer Disease and Associated Disorders, 21, 150-154. doi: 10.1097/WAD.0b013e318065c4a9

Brod, M., Steward, A. L., Sands, L. \& Walton, P. (1999). Conceptualization and measurement of quality of life in dementia: The Dementia Quality of Life instrument (DQoL). The Gerontologist, 39, 25-35. doi: 10.1093/geront/39.1.25

Castellón, A., Gómez, M. A. y Martos, A. (2004). Análisis de la satisfacción en los mayores de la Universidad de Granada. Revista Multidisciplinar de Gerontología, 14, 252-257.

Castellón, A. y Romero, V. (2004). Autovaloración de la calidad de vida. Revista Multidisciplinar de Gerontologia, 14, 131-137.

Diener, E. (1984). Subjective well-being. Psychological Bulletin, 95, 542-575. doi:10.1037/0033-2909.95.3.542

Felce, D. \& Perry, J. (1995). Quality of life: It's definition and measurement. Developmental Disabilities Research Reviews, 16 (1), 51-74. doi:10.1016/08914222(94)00028-8

Fernández-Merino, M. C., Vérez, L. y Gude, F. (1999). Morbilidad crónica y autopercepción de salud en los ancianos de una comunidad rural. Atencion Primaria, 17, 108-112.

Ferrando, J., Nebot, M., Borrell, C. y Egea, L. (1996). Apoyo social y estado de salud percibido en población no institucionalizada de más de 60 años. Gaceta Sanitaria, 10, 174-182. doi: 10.1016/S0213-9111(96)71893-9

Folstein M. F., Folstein S. \& Mchugh P.R. (1975). Mini-Mental State: A practical method for grading the cogntive state of patients for the clinicians. Journal of Psychiatric Research, 12 (3), 189-198. doi: 10.1016/00223956(75)90026-6

Hertzog, C., Kramer, A. F., Wilson, R.S. \& Lindenberger, U. (2008). Enrichment effects on adult cognitive development: Can the functional capacity of older adults be preserved and enhanced? Psychological Science in the Public Interest, 9 (1), 1-65. doi: 10.1111/j.1539-6053.2009.01034.x

Hoe, J., Katona, C., Orrell, M. \& Livingston, G. (2007). Quality of life in dementia: Care recipient and caregiver perceptions of quality of life in dementia: the LASER-AD study. International Journal of Geriatric Psychiatry, 22, 1031-1036. doi:10.1002/gps.1786
Hoe, J., Hancock, G., Livingston, G. \& Orrell, M. (2009). Changes in the quality of life of people with dementia living in care homes. Alz̧heimer Disease and Associated Disorders, 23(3), 285-290. doi: 10.1097/WAD.0b013e318194fc1e

Inouye, K. y Pedrazzani, E. S. (2007). Nivel de instrucción, socioeconómico y evaluación de algunas dimensiones de la calidad de vida de octogenarios. Revista Latino-Americana de Enfermagem, 15, 742-747. doi: 10.1590/S0104-11692007000700005

James, B. D., Xie, S. X. \& Karlawish, J. H. T. (2005). How do patients with Alzheimer disease rate their overall quality of life? The American Journal of Geriatric Psychiatry, 13, 484-90. doi: 10.1097/00019442-200506000-00007

Kitwood, T. \& Bredin, K. (1991). Towards a theory of dementia care: personhood and well-being. Ageing and Society, 12, 262-287. doi: 10.1017/S0144686X0000502X

Lapid, M. I., Rummans, T. A., Boeve, B. F., McCormick, J. K., Pankratz, V. S., Cha, RH. et al. (2011). What is the quality of life in the oldest old? International Psychogeriatrics, 52(3), 291-294. doi: 10.1017/ S1041610210002462

Lawton, M. P. (1994). Quality of life in Alzheimer disease. Alzheimer Disease and Associated Disorders, 8, 138-150.

Livingston, G., Cooper C., Woods, J., Milne, A. \& Katona, C. (2008). Successful ageing in adversity: The LASER-AD longitudinal study. Journal of Neurology, Neurosurgery, and Psychiatry, 79, 641-645. doi:10.1136/jnnp.2007.126706

Lobo, A., Ezquerra, J., Bugarda, F. G., Sala, J. M. y Seva, A. (1979). El MiniExamen Cognoscitivo. Un test sencillo y práctico para detectar alteraciones intelectuales en pacientes médicos. Actas Luso Españolas de Neurologia, Psiquiatría y Ciencias Afines, 7, 198-202.

Logsdon, R. G., Gibbons, L. E., McCurry, S. M. \& Teri, L. (1999). Quality of life in Alzheimer's disease: Patient and caregiver reports. Journal of Mental Health and Aging, 5, 21-32.

Logsdon, R. (2002). Assessing quality of life in older adults with cognitive impairment. Psychosomatic Medicine 64, 510-519.

Lopera, F. (1997). Clinical features of early-onset Alzheimer's disease in a large kindred with an E280A presenilin-1 mutation. JAMA, 277(10), 1104-1107. doi: 10.1001/jama.1997.03540340027028

Lövdén, M., Ghisletta, P. \& Lindenberger, U. (2004). Cognition in the Berlin Aging Study (BASE): The first 10 years. Aging, Neuropsychology, and Cognition, 11, 104-133.

Lucas-Carrasco, R. (2007). Calidad de vida y demencia. Medicina Clínica, 128, $70-75$.

Lucas-Carrasco, R., Pero, M. y March, J. (2011). Calidad de vida global en personas con demencia. Revista de Neurolología, 52 (3), 139-146.

Lucas-Carrasco, R., Skevington, S. M., Gómez-Benito, J., Rejas, J. \& March, J. (2011). Using the WHOQOL-BREF in patients with dementia: A Validation Study. Alzheimer Disease \& Associated Disorders 25(4), 345-51. doi: 10.1097/WAD.0b013e31820bc98b

Martínez-Martín P. (2004). Calidad de vida. Medicina Clínica, 5, 86-90.

Mckhann, G., Drachman, D. D., Folstein, M., Katsman, R., Price, D. \& Stadlen, E. M. (1984). Clinical Diagnoses of Alzheimer's disease: Report of the NINCDS-ADRDA Work Group under the auspices of the Department of Health and Human Services Task Force on Alzheimer's disease. Neurology, 34, 939-944. doi: 10.1212/WNL.34.7.939

Mehlsen, M., Kirkegaard-Thomsen, D., Viidik, A., Olesen, F. \& Zachariae, R. (2005). Cognitive processes involved in the evaluation of life satisfaction: Implications for well-being. Aging and Mental Health, 9 (3), 281290. doi: 10.1080/13607860412331310236

Missotten, P., Ylieff, M., Di Notte, D., Paquay, L., De Lepeleire, J., Buntinx, F. \& Fontaine, O. (2007). Quality of life in dementia: A 2-year follow up study. International Journal of Geriatric Psychiatry, 12, 1201-1207. doi: 10.1002/gps.1814

Molina, C., Meléndez, J. C. y Navarro, E. (2008). Bienestar y calidad de vida en ancianos institucionalizados y no institucionalizados. Anales de Psicologia, 24, 312-319. doi:10.6018/analesps.24.2.42861

Nagamoto, I., Kita, K., Takigawa, M., Nomaguchi, M. \& Sameshima, K. (1997). A study of the quality of life in elderly people using psychological testing. International Journal of Geriatric Psychiatry, 12, 599-608. doi: 
10.1002/(SICI)1099-1166(199706)12:6<599::AID-GPS505>3.0.CO;2E

Organización Mundial de la Salud. Programa Envejecimiento y Ciclo Vital (2002). Envejecimiento activo: Un marco político. Revista Española de Geriatría y Gerontología, 37(S2),74-105.

Oswald, W. (2004). Cognitive and physical activity - A way for maintaining independent living and delaying the onset of dementia? European Review of Aging and Physical Activity, 1, 49-59.

Rabins, P. V. \& Black, B. S. (2007). Measuring quality of life in dementia: purposes, goals, challenges and progress. International Psychogeriatrics, 19, 401-407. doi:10.1017/S1041610207004863

Ramírez, R., Agredo, R. A., Jerez, A. M., y Chapal, L. Y. (2008). Calidad de vida y condiciones de salud en adultos mayores no institucionalizados en Cali, Colombia. Revista de Salud Pública, 10 (4), 529-536.

Ready, R. E., Ott, B. R. \& Grace, J. (2006). Insight and cognitive impairment: effects on quality-of-life reports from mild cognitive impairment and Alzheimer's disease patients. American Journal of Alzheimer's Disease and Other Dementias, 21, 242-248. doi: 10.1177/1533317506290589

Revell, A. J., Caskie, G., Willis, S. L. \& Schaie, K. W. (2009). Factor structure and invariance of the quality of life in Alzheimer's disease (qol-ad) scale. Experimental Aging Research, 35(2), 250 -267.

Rodríguez, M. y Sanchez, J. L. (2004). Reserva cognitiva y demencia. Anales de Psicología, 20, 175-186. doi:10.6018/analesps.20.2.2730

Sánchez, J. L., Torrellas, C., Martín, J. \& Barrera, I. (2011) Study of sociodemographic variables linked to lifestyle and their possible influence on cognitive reserve. Journal of Clinical and Experimental Neuropsychology, 33(8), 874-891. doi: 10.1080/13803395.2011.567976

Schiffczyk, C., Romero, B., Jonas, C. \& Riepe, M. (2010). Generic quality of life assessment in dementia patients: A prospective cohort study. BMC Neurology, 10, 48. doi: 10.1186/1471-2377-10-48

Séculi, E., Fusté, J., Brugulat, P., Juncá, S., Rué, M. \& Guillén, M. (2001). Percepción del estado de salud en varones y mujeres en las últimas etapas de la vida. Gaceta Sanitaria, 15 (3), 217-23. doi: 10.1016/S02139111(01)71550-6

Selai, C., Trimble, M., Rossor, M. \& Harvey, R. (2001) Assessing quality of life in dementia. Preliminary psychometric testing of the Quality of Life Assessment Schedule (QOLAS). Neuropsychological Rehabilitation, 1, 219243.

Selwood, A., Thorgrimsen, L. \& Orrell, M. (2005). Quality of life in dementia - a one year follow-up study. International Journal of Geriatric Psychiatry, 20, 232-237. doi: 10.1002/gps.1271

Schwartzmann, L. (2003). Calidad de vida relacionada con la salud: Aspectos conceptuales. Revista Ciencia y Enfermería, 9 (2), 9-21. doi: 10.4067/S0717-95532003000200002

Sloane, P. D., Zimmerman, S., Williams, C. S., Reed, P. S., Gill, K. S. \& Preisser, J. S. (2005). Evaluating the quality of life of long-term care residents with dementia. The Gerontologist, 45, 37-49. doi: 10.1093/geront/45.suppl_1.37

Smith, S. C., Lamping, D. L., Banerjee, S., Harwood, R., Foley, B., Smith, P., et al. (2005). Measurement of health-related quality of life for people with dementia: Development of a new instrument (DEMQOL) and an evaluation of current methodology. Health Technology Assessment, 9, 1-93.

Stern, Y. (2002). What is cognitive reserve? Theory and research application of the reserve concept. Journal of the International Neuropsychological Society, 8, 448-460. doi: 10.1017.S1355617701020240

Thorgrimsen, L., Selwood, A., Spector, A., Royan, L., de Madariaga-Lopez, M., Woods, R.T. \& Orrell, M. (2003). Whose quality of life is it anyway? The Validity and Reliability of the Quality of Life - Alzheimer's Disease (QOL-AD) Scale. Alsheimer Disease \& Associated Disorders, 17, 201-208.

Valenzuela, M. J. (2008). Brain Reserve and the prevention of dementia. Current Opinion in Psychiatry, 21, 296-302. doi: 10.1097/ YCO.0b013e3282f97b1f

Velarde-Jurado, E. y Ávila-Figueroa, C. (2002). Evaluación de la calidad de vida. Salud Pública de México, 44, 349-361.

Vilagut, G., Valderas, J. M., Ferrer, M., Garin, O., López-García, E. y Alonso, J. (2008). Interpretación de los cuestionarios de salud SF-36 y SF-12 en España: Componentes físico y mental. Medicina Clinica, 130 (19), 726-735.

Vilagut, G., Ferrer, M., Rajmil, L., Rebollo, P., Permanyer-Miralda, G., Quintana, J.M. et al. (2005). The Spanish version of the Short Form 36 Health Survey: A decade of experience and new developments. Gaceta Sanitaria, 19(2), 135-150. doi: 10.1590/S0213-91112005000200007

Vogel, A., Stokholm, J., Gade, A., Andersen, B. B., Hejl, A. M. \& Waldemar, G. (2004). Awareness of deficits in mild cognitive impairment and Alzheimer's disease: Do MCI patients have impaired insight? Dementia and Geriatric Cognitive Disorders, 17, 181-187. doi: 10.1159/000076354

Waldorff, F.B., Nielsen, A.B. \& Waldemar, G. (2010). Self-rated health in patients with mild Alzheimer's disease: Baseline data from the Danish Alzheimer Intervention Study. Archives of Gerontology and Geriatrics, 50, 1 5. doi:10.1016/j.archger.2008.12.010

Ware, J. E. \& Sherbourne, C. D., (1992). The MOS 36-item short form health survey (SF-36): Conceptual framework and item selection. Medical Care, 30, 473-83.

Whitehouse, P. (1999). Quality of life in Alzheimer's disease: Future directions. Journal of Mental Health and Aging, 5, 107-110.

Wierenga, C. E \& Bondi, M. W (2007). Use of functional magnetic resonance imaging in the early identification of Alzheimer's disease. Neuropsychology Review, 17, 127-143. doi: 10.1007/s11065-007-9025-y

Woods, B., Thorgrimsen, L., Spector, A., Royan, L. \& Orrell, M. (2006). Improved quality of life and cognitive stimulation therapy in dementia. Aging and Mental Health, 10 (3), 219-226. doi: 10.1080/ 13607860500431652

Zea-Herrera, M. C., López, M. E., Valencia, C. M., Soto, J. A., Aguirre, D. C., Restrepo, F. L., Oswald, W. D. y Rupprecht, R. (2008). Autovaloración de calidad de vida y envejecimiento en adultos con riesgo de Alzheimer. Investigación y Educación en Enfermería, 26 (1), 24-35.

Zúniga, M. A., Carrillo, G. T., Fos, P. J., Gandek, B. y Medina-Moreno, M.R. (1999). Evaluación del estado de salud con la Encuesta SF-36: Resultados preliminares en México. Salud Pública de México, 41, 110-118. doi: 10.1590/S0036-36341999000200005

(Artículo recibido: 17-10-2011; revisado: 27-9-2012; aceptado: 20-10-2012) 